Apidologie, 1977, 8 (1) 61-82.

\title{
POSSIBILITÉ DE MULTIPLICATION DU POLLINISATEUR DE LA LUZERNE MEGACHILE PACIFICA PANZ. EN FRANCE
}

\author{
Über die Möglichkeit, den Luzerne-Bestäuber Megachile pacifica (Panz.) \\ in Frankreich zu vermehren
}

J.-N. TASEI

avec la collaboration technique de J. ALETRU, M. HOUILLIER, CH. LACROIX, M. LATIL, J. LEMENAHEze, P. PAGÈs

Laboratoire de Zoologie, I.N.R.A., 86600 Lusignan

SUMMARY

POSSIBILITY OF POPULATION INCREASE

in FRANCE FOr the LUCERne POllinator :

Megachile pacifica Panz.

This article reports encouraging results for leafcutter bee Megachile pacifica field management in 1975. The first generation flight occured in July, the second in August and September. In spite of low levels of food plants and low temperatures unfavourable to second generation activity, the average multiplication rate of cells was $1: 1,7$ in 11 experimental standard shelters distributed in South, South-East, Middle West France.

Losses due to parasites, predators (Melittobia, Miltogramma, Trichodes), etc. reached 22 per cent of the cells. Female ratio of the progeny $(0,8-1)$ was higher than that of the parental population $(0,4-1)$. As a consequence the female increase rate was $1: 2,2$ on average.

Three out of 11 shelters were encased in polyethylene. This increases the attractiveness of nesting sites, and seems to be a promising technique.

Studies on the diapause, the French megachile univoltine strain and parasite dynamics will be carried out.

\section{RESUME}

Cet article fait part de résultats encourageants concernant l'élevage en champ de l'abeille Megachile pacifica en 1975. La première génération vole en juillet, la seconde en août et septembre. En dépit de la rareté des plantes nourricières et d'une température basse qui ne favorisent pas l'activité de la seconde génération, le taux moyen de multiplication des cellules 
est $1: 1,7$ dans 11 abris expérimentaux identiques implantés dans le Sud, Sud-Est, et CentreOuest de la France.

Les pertes dues aux parasites, prédateurs (Melittobia, Miltogramma, Trichodes), etc. affectent 22 pour cent des cellules. Dans la progéniture la proportion des femelles $(0,8-1)$ est plus élevée que dans la population parentale $(0,4-1)$. En conséquence le taux de multiplication des femelles est en moyenne $1: 2,2$.

Trois des 11 abris sont en polyane. Ce procédé qui accroît l'attractivité des sites de nidification semble être prometteur.

Des études sur la diapause, sur la souche monovoltine de mégachile français et sur la dynamique des populations de parasites seront entreprises.

\section{INTRODUCTION}

Les difficultés rencontrées localement dans notre pays en production grainière de luzerne sont en partie liées, soit à la rareté de la faune pollinisatrice spécifique, représentée par quelques espèces d'abeilles sauvages, soit à la mauvaise coïncidence de leur vol avec la période de floraison. La pollinisation de la luzerne peut être assurée de manière régulière et rationnelle à condition de maîtriser parfaitement le cycle d'au moins une espèce d'apoïde. Megachile pacifica, abeille solitaire paléarctique est une des deux espèces domestiquées par des chercheurs américains (BонART, 1962) et élevées dans les luzernières pour améliorer les rendements grainiers. Ce mégachile, de maniement relativement simple est élevé au Canada (Hoвbs, 1965), au Chili (Anonyme, 1972), en Tchécoslovaquie (PтAcek, 1975), en Hongrie (Manninger, 1975) en Roumanie (Ciurdarescu et Varga, 1975) et depuis 1972 en France (TASEI, 1975).

Au cours des 3 premières années d'étude qui se sont déroulées dans le Sud-Est du pays, les techniques utilisées n'ont pas permis de multiplier la population initiale. En effet divers facteurs ont conjugué leur action et contrarié la réussite espérée, principalement :

- Le manque d'attractivité des nichoirs proposés.

- Les pertes dues à une abondante deuxième génération émergeant après le retrait hors du champ des sites de nidification.

-- Les dégâts occasionnés par Trichodes apiarius coléoptère prédateur des stades immatures :

La confrontation de certaines observations a mis en évidence l'importance de certains détails dans la construction des nichoirs et dans les manipulations des cocons. Par ailleurs, on a vu que la deuxième génération pouvait être élevée dans le champ. Par conséquent, en 1975, on s'efforce d'appliquer les techniques considérées comme étant les plus sûres et aussi les plus simples. Cette mise à l'épreuve a lieu dans 11 abris identiques implantés dans plusieurs régions productrices de graine de luzerne. 


\section{MATÉRIEL ET METHODES}

\section{1. - Les lieux d'élevage}

La figure 1 indique l'emplacement de 8 localités du Centre-Ouest, Sud et Sud-Est près desquelles on installe 11 abris à mégachiles dans 9 champs (tabl. 1). Tous les champs ont fleuri pendant le mois de juillet. La pleine floraison est assez variable. A titre indicatif certains champs possèdent un maximum de 200 inflorescences épanouies au mètre carré, d'autres 400.

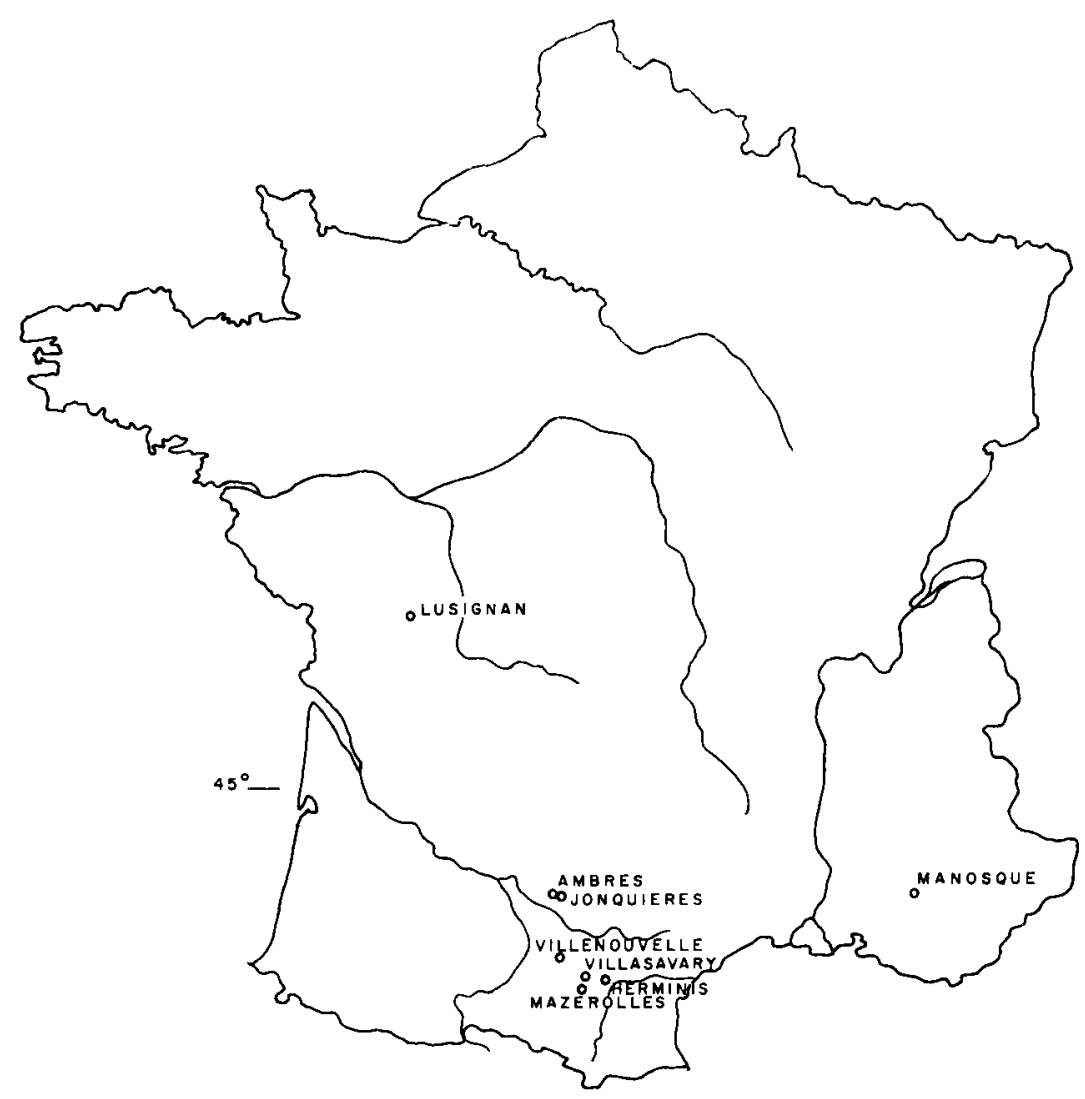

FIG. 1. - Emplacement des champs de luzerne où l'on a installé des sites à mégachiles en 1975.

Aвв. 1. - Lage der Luzernefelder, in denen 1975 die Nistanlagen für Megachile aufgestellt waren.

Pendant la période de vol des mégachiles les seuls champs traités aux insecticides sont : Manosque (2 traitements), Ambres (1), Jonquières (3). Tous ces traitements sont effectués avec de la "Phosalone ", exception faite du troisième traitement de Jonquière (pulvérisation de " Phosalone » + ( Endosulfan "). 
Tаві. 1.-- Dimensions des champs et répartition des sites de nidification à mégachiles en 1975.

'Тав. 1. -- Grösse der Felder und Verteilung der Nistanlagen für Megachile, 1975.

\begin{tabular}{|c|c|c|}
\hline $\begin{array}{c}\text { Lien } \\
\text { Ort }\end{array}$ & $\begin{array}{c}\text { Surface du champ } \\
\text { en hectares } \\
\text { Gröse de's Feldes } \\
\text { in ha }\end{array}$ & $\begin{array}{l}\text { Nombre d'abris } \\
\text { Zahal der Schut } \% \\
\text { häuschen }\end{array}$ \\
\hline Sud-Est (Alpes de Haute Provence) & & \\
\hline Manosque .. & 10 & $\mathbf{l}$ \\
\hline Sud (Aude - Tarn - Haute-(Garonne) & & \\
\hline Herminis........ & 4. & 1 \\
\hline Mazerolles................. & 7 & 2 \\
\hline Villasavary $\ldots \ldots \ldots \ldots \ldots$. & 1 & 1 \\
\hline Villenouvelle .......... & 15 & l \\
\hline Ambres $\ldots \ldots \ldots \ldots \ldots \ldots \ldots$ & 6 & 1 \\
\hline Jonequières ........... & 8 & 1 \\
\hline Centre-ouest (Vienne) & & \\
\hline Lusignan. . . . & 0.5 & 2 \\
\hline Lusignan ...... & 0,03 & 1 \\
\hline
\end{tabular}

2. - Les dispositifs de nidification

Le dispositif standard (fig. 2) est composé :

- D'un abri en contreplaqué peint en jaune $(0,70 \times 0,55 \times 0,30 \mathrm{~m})$ muni d'un toit incliné. Des piquets de fer latéraux et des haubans assurent l'immobilité de l'ensemble orienté vers le Sud.

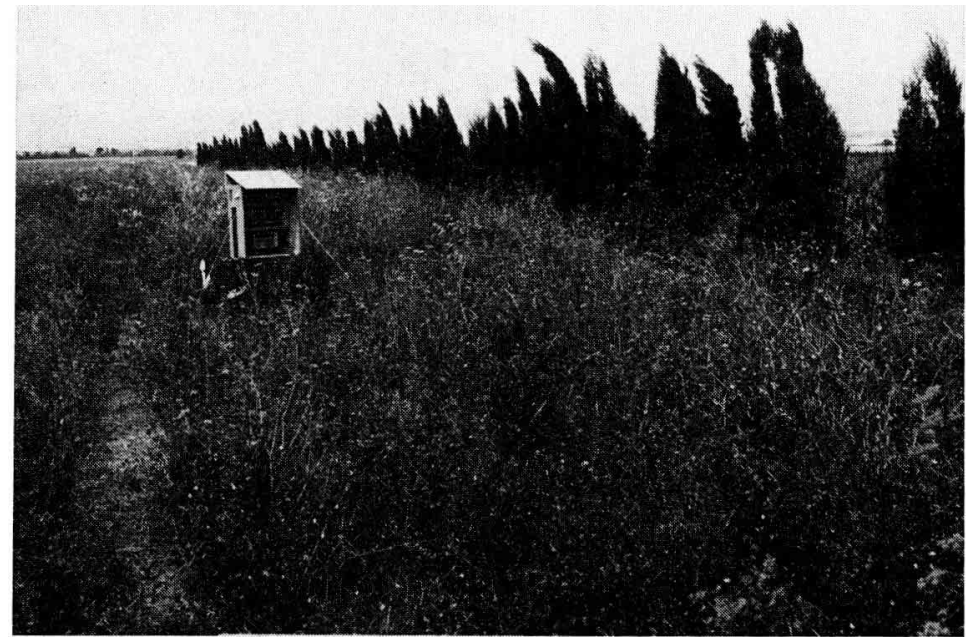

Fıg. 2. - Abri à mégachiles dans une luzernière.

Aв8. 2. - Schutzäuschen für die Megachile - Nistanlagen in einem Luzernefeld. 
- D'un nichoir en bois de Pin, à plaques cannelées offrant 880 trous de $5 \mathrm{~mm}$ de diamètre et $8 \mathrm{~cm}$ de longueur. Le fond des trous est obturé par une feuille de caoutchouc collée sur la plaque vissée qui fait office de dos.

- D'un " éclosoir » renfermant les cellules "parents » accroché au-dessus du nichoir.

- D'un pondoir à Trichodes installé à la partie inférieure. Il s'agit d'un empilement lâche de plaques de bois à grosses cannelures ( $8 \mathrm{~mm}$ de diamètre) au fond desquelles on dispose un mélange sirupeux de pollen et d'eau sucrée.

Une variante du dispositif est un abri en polyane placé au-dessus de l'abri en contreplaqué (fig. 3). Ce dispositif est testé en 2 endroits : Mazerolles (1 abri), Lusignan (2 abris).

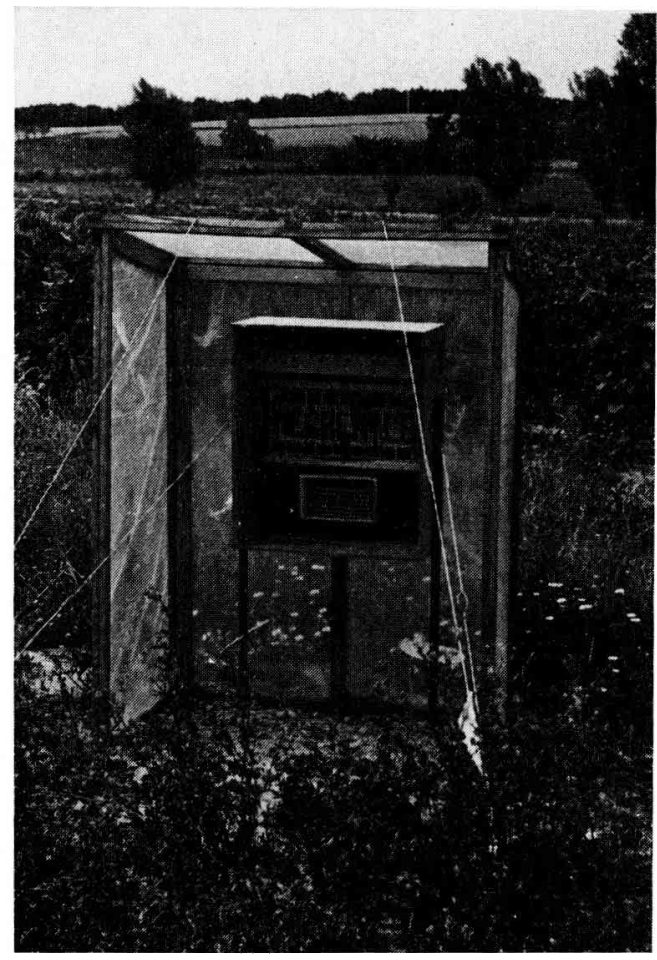

FIG. 3. - Abri à mégachiles en polyane

Авв. 3. - Polyäthylen-Schutzhäuschen.

\section{3. - La préparation et l'installation des abeilles dans le champ}

On réactive dans une étuve réglée à $29^{\circ} \mathrm{C}$ un total de 23600 cellules importées du Canada dont la proportion des sexes est 27 femelles pour 73 mâles. L'étuve possède une lampe à ultraviolet a fin de pièger les parasites pouvant sortir des cocons. Dès que les premiers mâles apparaissent on installe dans les éclosoirs 2300 à 2500 cocons par abri, sauf à Lusignan où I'on dispose respectivement dans les 3 abris de 1900 , 1800 à 900 cellules environ. L'implantation des nichoirs a lieu vers le 5 juin pour les sites de l'Aude et de Lusignan, 3 semaines plus tard pour tous les autres. Les abris restent dans le champ le temps nécessaire à la construction des cellules de deuxième génération. 


\section{4. - Les contrôles}

Une fois par semaine chaque abri est visité : on compte le nombre de nids bouchés et à partir du mois d'août le nombre de nids débouchés. A Lusignan les observations sont plus fréquentes et on compte de façon précise le nombre de femelles au repos dans les tubes.

Lorsque la deuxième génération a cessé de construire, les nichoirs sont ramenés au laboratoire, et démontés, ce qui permet de compter les cellules et les déprédateurs les plus visibles (larves de Trichodes et pupes de Miltogramma). Pour chaque abri on ouvre 300 cellules apparemment saines a fin d'estimer la mortalité larvaire et de déterminer les parasites pouvant se trouver à l'intérieur des cocons. On contrôle aussi la mortalité en " éclosoir " sur 500 cellules. Le sex ratio de chacun des nichoirs est évalué à partir d'une centaine de cellules qui sont réactivées à $29^{\circ} \mathrm{C}$ au mois de janvier, après 2 à 3 mois d'hibernation à 5 -- $10^{\circ} \mathrm{C}$.

\section{RÉSULTATS}

\section{I. - Accroissement de la population dans les sites de nidification}

Le tableau 2 donne les rapports de multiplication des cellules avant et après déduction des pertes diverses. Ces rapports varient respectivement de 0,80 à 2,32 et de 0,48 à 2 . Si l'on tient compte de la proportion de femelles (détail sur le tabl. 3) qui est en moyenne de 46 pour cent, les rapports de multiplication des femelles sont plus élevés (de 0,4 à 3,4 ) puisqu'il il y a eu augmentation de la proportion des femelles au cours de la saison d'élevage. La majorité des abris fournit au moins 2 fois plus de femelles qu'il n'y en avait dans l’éclosoir.

TaBl. 2. - Accroissement de la population de Megachile pacifica dans 11 sites de nidification en 1975.

TAв. 2. - Zunahme der Populationen von Megachile pacifica an elf Nistplätzen, 1975.

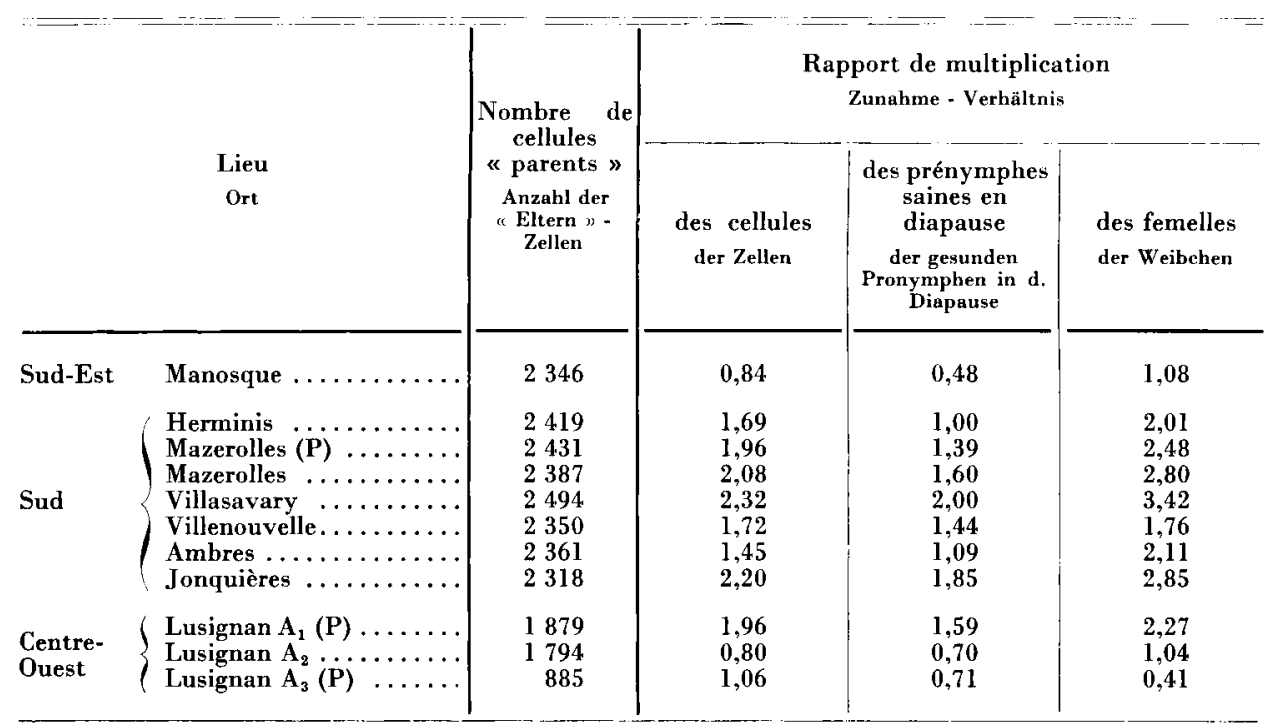

(P) = nichoirs sous abri en polyane.

(P) = Polyäthylen-Schutzhäuschen. 


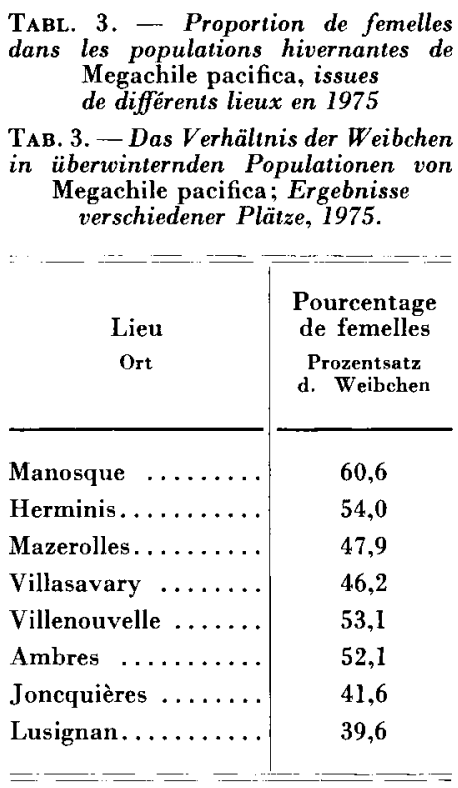

TABL. 4. - Nombre d'heures d'activitê des Mégachiles en 1975.

TAB. 4. - Anzahl der Arbeitsstunden von Megachile, 1975.

\begin{tabular}{|c|c|c|}
\hline \multirow{2}{*}{$\begin{array}{l}\text { Lieu } \\
\text { Ort }\end{array}$} & \multicolumn{2}{|c|}{$\begin{array}{c}\text { Nombre d'heures chaudes * (température supérieure à } 24^{\circ} \mathrm{C} \text { ) } \\
\text { Anzahl warmer Stunden * (Temperaturen höher als } 24{ }^{\circ} \mathrm{C} \text { ) }\end{array}$} \\
\hline & $\begin{array}{c}\text { 1er juillet au } 31 \text { juillet } \\
=\text { vol de } 1^{\text {re }} \text { génération } \\
\text { 1. Juli bis } 31 \text {. Juli } \\
=\text { Flug d. ersten Generation }\end{array}$ & $\begin{array}{l}15 \text { août au } 10 \text { octobre } \\
=\text { vol de } 2 \text { e génération } \\
\text { 15. August bis } 10 \text {. Oktober } \\
=\text { Flug der zweiten Generation }\end{array}$ \\
\hline Manosque $\ldots \ldots \ldots \ldots \ldots \ldots \ldots \ldots$ & 252 & 245 \\
\hline 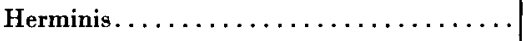 & 238 & 178 \\
\hline Mazerolles . . . . . . . . . . . . . . . & 245 & 122 \\
\hline Villasavary $\ldots \ldots \ldots \ldots \ldots \ldots \ldots \ldots \ldots$ & 252 & 188 \\
\hline Villenouvelle $\ldots \ldots \ldots \ldots \ldots \ldots \ldots \ldots$ & 203 & 122 \\
\hline $\left.\begin{array}{l}\text { Ambres } \\
\text { Jonquières }\end{array}\right\} \ldots \ldots \ldots \ldots \ldots \ldots \ldots$ & 259 & 178 \\
\hline Lusignan . . . . . . . . . . . . . . . . & 118 & 49 \\
\hline
\end{tabular}

* Heures comprises entre 8 heures du matin et 7 heures du soir.

* Die Zeit zwischen 8 und 19 Uhr. 
Les variations de multiplication d'un lieu à un autre ne peuvent être expliquées par l'action directe des facteurs climatiques (tabl. 4). En effet l'endroit le plus chaud de juillet à octobre (Manosque) a donné les moins bons résultats tandis qu'à Lusignan où le climat est le plus tempéré, le meilleur des résultats égale ou dépasse ceux de localités méridionales nettement plus chaudes.

Les figures 4,5 et 6 montrent quelle est approximativement la dynamique de la population larvaire au cours des vols de première et deuxième génération pour chacun des 10 abris représentés. On observe une première phase de remplissage du nichoir durant environ un mois $(1$ mois et demi à Lusignan) puis une diminution plus ou moins bruta'e du nombre de nids, due à l'émergence de la deuxième génération pendant 1 mois. La troisième phase est le nouveau remplissage du nichoir par les femelles de deuxième génération durant 3 à 4 semaines. On note qu'en fin de saison (début octobre) la population larvaire est sensiblement inférieure à celle qui est issue de la première génération début août, malgré la présence d'un nombre de femelles plus important pendant la deuxième génération que pendant la première, comme cela peut être observé à Lusignan dans l'abri sous polyane (fig. 6). Cette faible nidification est liée aux basses températures de fin d'été (tabl. 4) qui ne permettent pas aux femelles de voler longtemps chaque jour, et qui réduisent leur longévité.

\section{II. - Analyse des résultats}

\section{La mortalité dans la population parent}

La mortalité chez les individus parents contenus dans les éclosoirs est de l'ordre de 10 pour cent $( \pm 0,8)$. Elle affecte 2 fois plus les prénymphes que les nymphes. Quatre vingt dix pour cent des individus sains émergent dans les 2 semaines suivant l'installation dans le champ.

\section{Le retour au site d'émergence. L'attractivité des nichoirs et des abris}

Dans 8 sites sur 11 , plus de 50 pour cent des femelles qui sortent des éclosoirs reviennent sur place pour nidifier. A Manosque et dans 2 des 3 sites établis à Lusignan une forte proportion des femelles n'est pas revenue au nichoir pour des raisons indéterminées.

\section{La deuxième génération}

Les émergences des individus qui ne restent pas à l'état de larves en diapause, sont assez importantes puisqu'elles concernent 44 à 77 pour cent des nids construits par les femelles de première génération en juillet. Les 

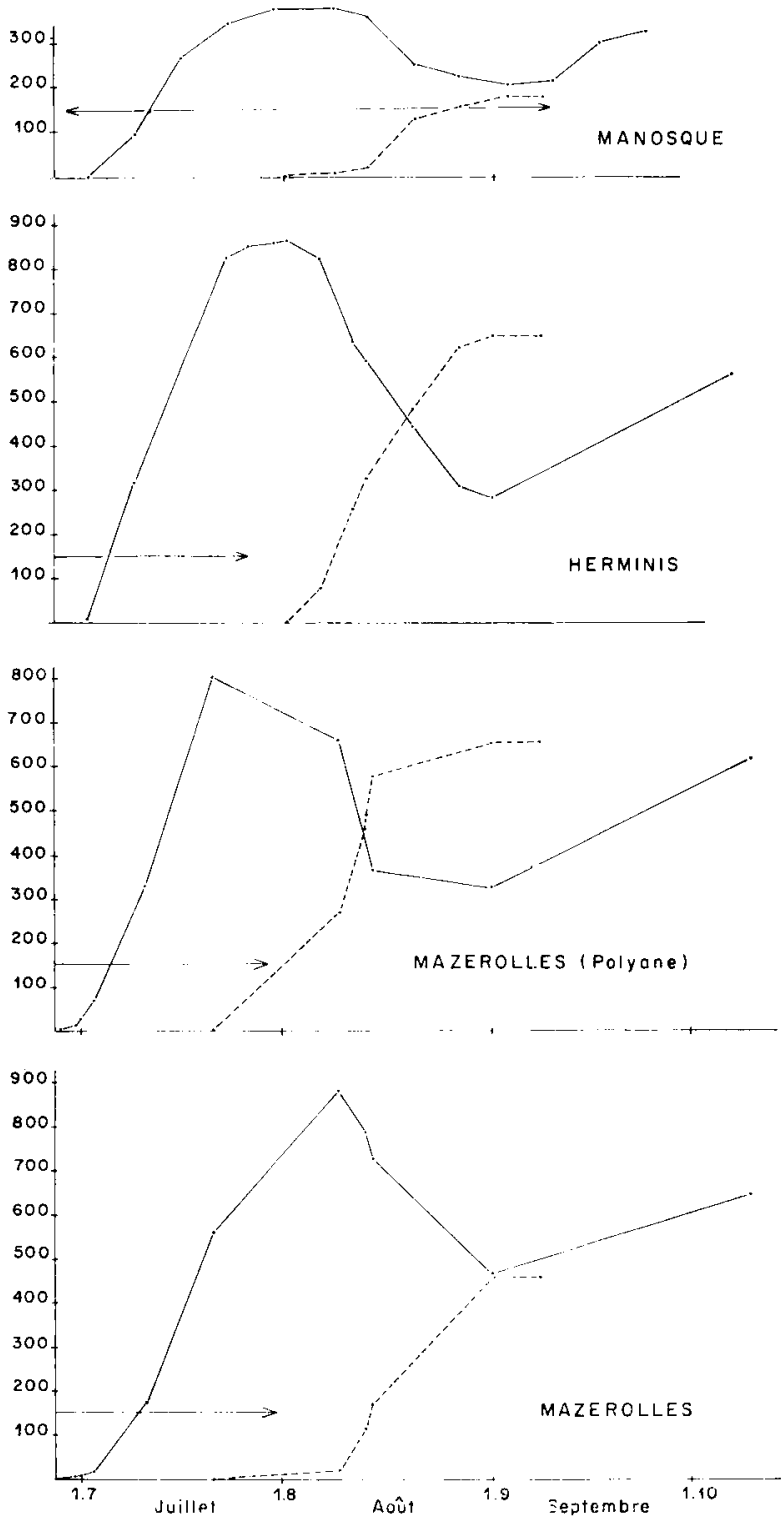

Fig. 4. - Dynamique de la population des larves à l'intérieur des nichoirs dans 4 abris en 1975. Verticalement : nombre de nids

$-$\begin{tabular}{l} 
nids terminés \\
\hline$-\ldots+\ldots$
\end{tabular} $\longleftrightarrow \quad$ : floraison de la luzerne

Aвв. 4. - Die Dynamik der Maden-Populationen im Innern Ordinate $=$ Zahl der Nester der Nistanlagen vier verschiedener Schutzhäuschen; 1975.

$\underline{-}=$ fertig gebaute Nester

$--_{-}=$durch den Ausflug der 2. Generation geöffnete Nester

$\longleftrightarrow=$ Blütezeit der Luzerne 

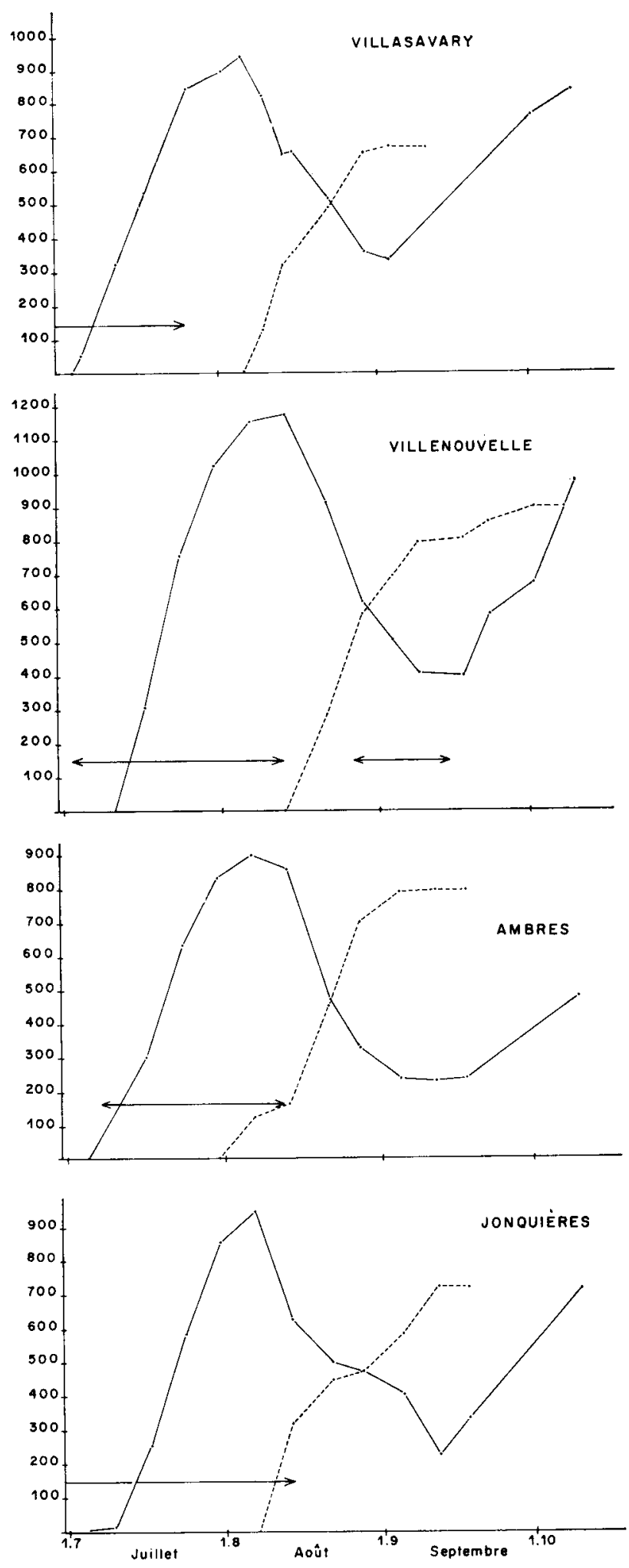

Fig. 5. - Même légende que figure 4.

Aвв. 5. - Wie Abb. 4. 
pourcentages les moins élevés sont observés à Lusignan, Manosque et Mazerolles, lieux dont les températures nocturnes sont les plus basses. Dans les 3 abris sous polyane les émergences sont plus nombreuses que dans les abris comparables de type ordinaire.

\section{L'activité de nidification des femelles}

Notons tout d'abord, que dans plusieurs endroits le remplissage des nichoirs par les femelles s'est effectué très rapidement en début de saison. Les 880 trous disponibles de chaque nichoir ont tous été occupés et de nombreuses femelles, entrant en compétition pour l'habitat, se sont mutuellement gênées. Cette gêne s'est parfois manifestée par des destructions de nids, les trous ainsi dégagés étant occupés à nouveau.

Les températures du mois de juillet sont partout assez élevées (tabl. 4) et favorisent l'activité des femelles de première génération. De même les températures du début du mois d'août favorisent les sorties de la deuxième génération, tandis que les températures du mois de septembre sont très basses pour la saison et ne sont pas favorables à l'activité des femelles de deuxième génération. A Lusignan pendant la première décade de septembre, il n'y a pas de température supérieure à $23^{\circ} \mathrm{C}$ et pendant la deuxième décade il n'y a pas de température supérieure à $22^{\circ} \mathrm{C}$. Malgré cette entrave à l'activité des mégachiles, quelques nids sont terminés dans les abris $A_{1}$ et $A_{2}$ (fig. 7).

On peut constater sur les figures 4 et 5 que la floraison de la luzerne se prolonge parfois début août et très rarement en septembre. Elle profite ainsi à la première génération mais pratiquement pas à la deuxième. Le tableau $\mathbf{n}^{\circ} 5$ indique quelles sources de pollen les femelles de deuxième génération sont allées butiner. Les plantes qui malgré leur faible abondance ont permis la survie des adultes et l'approvisionnement des nids en août-septembre sont surtout les Composées (Helminthia, Inula, Picris) et moins fréquemment des Crucifères, des Ombellifères, des Rosacées (Rubus) et des Papilionacées (Lotus).

On estime qu'une femelle de première génération a construit selon le lieu d'élevage de 15 à 20 cellules, tandis qu'une femelle de deuxième génération en a construit seulement 2 ou 3 . Trois raisons peuvent être invoquées pour expliquer cette différence : les températures anormalement basses fin août et début septembre; les jours plus courts; la rareté et l'éloignement des sources de nectar et de pollen après la floraison de la luzerne.

\section{Les pertes observées dans la progéniture}

Le tableau no 6 récapitule les types d'attaque selon l'espèce de prédateur ou parasite et selon le lieu d'implantation des abeilles. Certains sites sont l'objet de peu de destructions ( 1 à 5 pour cent) : Villasavary, Villenouvelle, 

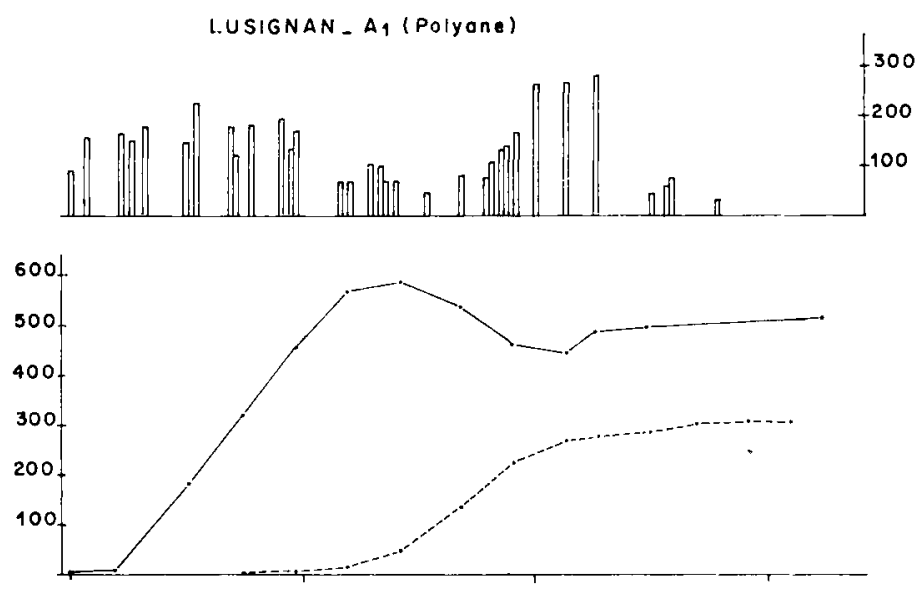

LUSIGNAN_A2
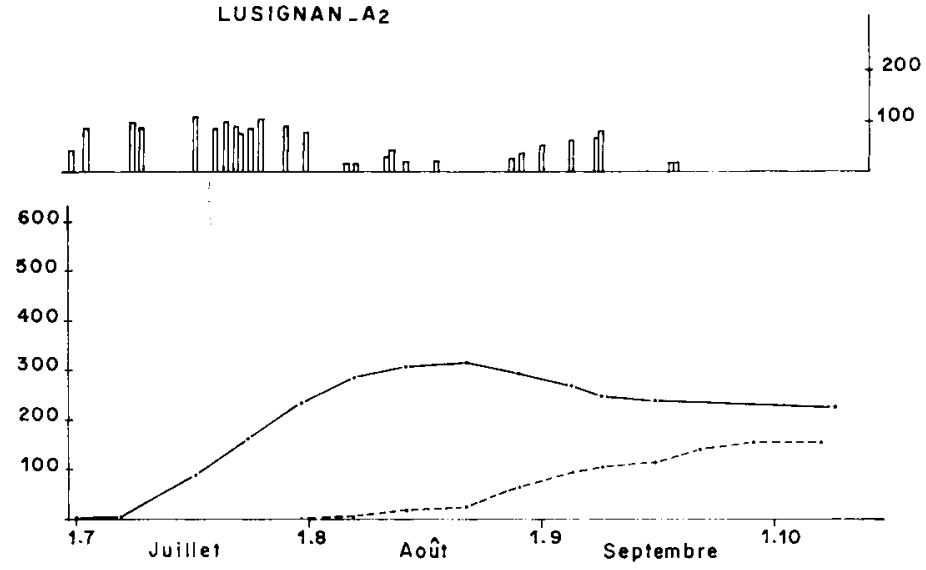

FiG. 6. - Dynamique de la population des femelles actives et des larves à l'intérieur des nichoirs de 2 abris à Lusignan en 1975. (Populations parentales identiques.)

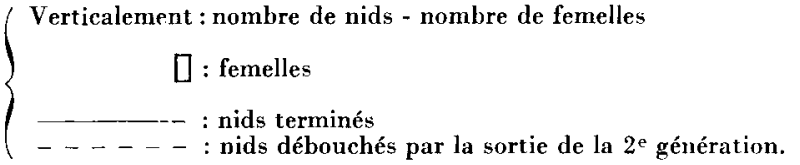

AB8. 6. - Dynamik der aktiven Weibchen-Population und der Maden im Innern von zwei Nistanlagen; Lusignan 1975 (Identische « Eltern »-Populationen).

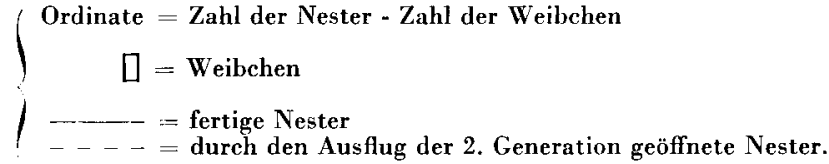




\section{LUSIGNAN_A1 (PO!YORE)}
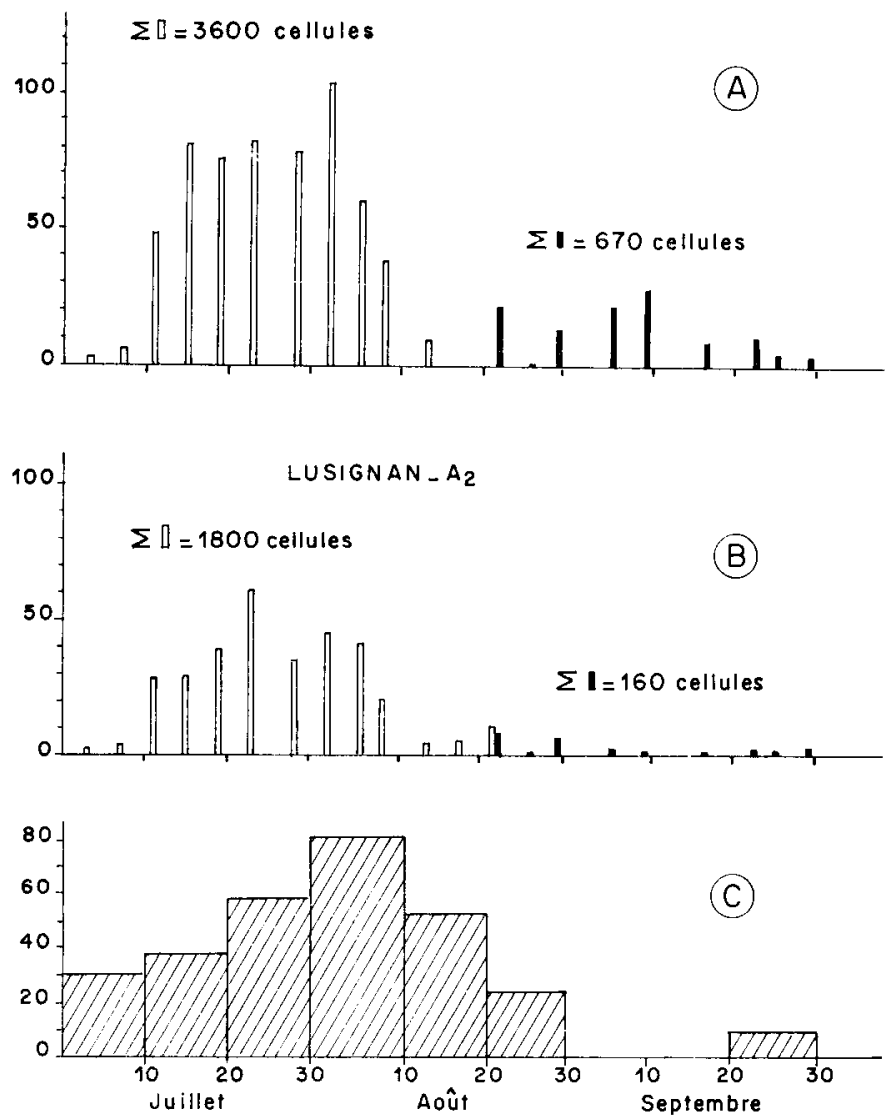

FIg. 7. - Activitê de nidification et températures dans 2 abris à Lusignan en 1975.

Verticalement : A et B : Nombre de nids

C : Nombre d'heures par décade.

口 : Nids construits par des femelles de $1^{\text {re }}$ génération

: Nids construits par des femelles de $2^{\mathrm{e}}$ génération

$\mathbb{Z}$ : heures chaudes (température $>24^{\circ} \mathrm{C}$ ).

Авв. 7. - Nestbautätigkeit und Temperaturen in zwei Nistanlagen in Lusignan 1975.

Ordinate $=\mathrm{A}$ und $\mathrm{B}:$ Zahl der Nester

C : Zahl der Stunden je Dekade

$\square=$ von den Weibchen der 1. Generation gebaute Nester

= von den Weibchen der 2. Generation gebaute Nester

$\mathbb{Z}=$ Anzahl Stunden mit hohen Temperaturen (höher als $24{ }^{\circ} \mathrm{C}$ ) 
TABL. 5. - Pollens récoltés en août-septembre 1975 par Megachile pacifica dans 8 champs, après la floraison de luzerne.

TAB. 5. - Vom Megachile pacifica im August/September 1975 nach der Blütezeit der Luzerne in acht Feldern gesammelter Pollen.

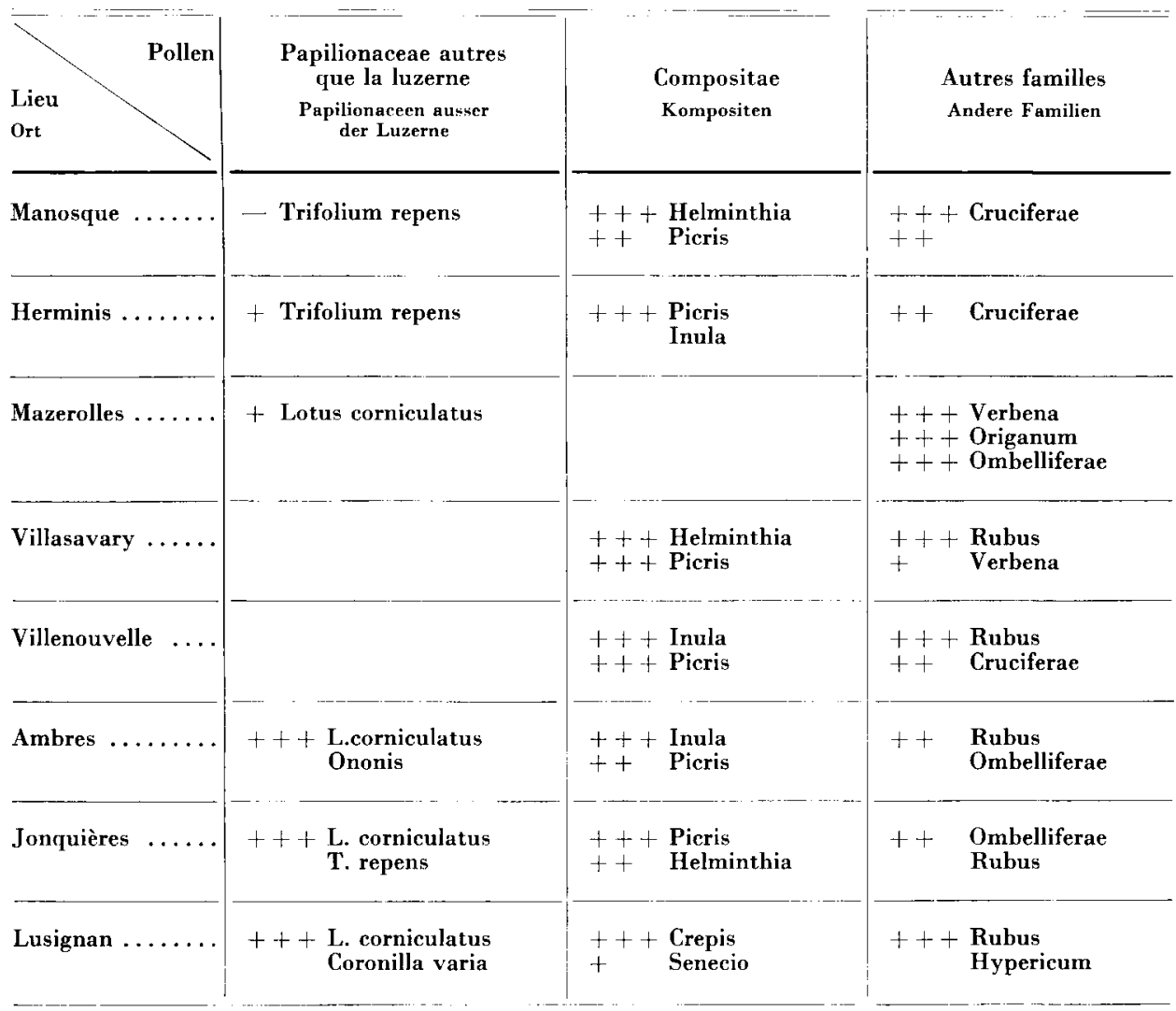

\footnotetext{
$-=1$ à $10 \%$ ～environ de la masse des pollens autres que le pollen de luzerne

$+=10 \%$
$++=20 \%$$\quad\{$ ungefähr der Pollenmenge ausser dem Luzernepollen
}

Jonquières, Lusignan $A_{2}$. En général les trois ravageurs principaux sont : Trichodes apiarius L. (Coléoptère Cleridae), Miltogramma murinum Mg. (Diptère Tachinidae), Melittobia acasta Walk. (Hyménoptère Eurytomidae). Chez les stades immatures la mortalité pour cause indéterminée (mycose, virus, intoxication, accident physiologique du à l'excès de chaleur, etc.) varie de 10 à 17 pour cent. 
TABL. 6. - Ennemis de Megachile pacifica. Taux d'attaque suivant le lieu en 1975.

Tав. 6. - Feinde von Megachile pacifica. Befallsrate je nach Ort; 1975.

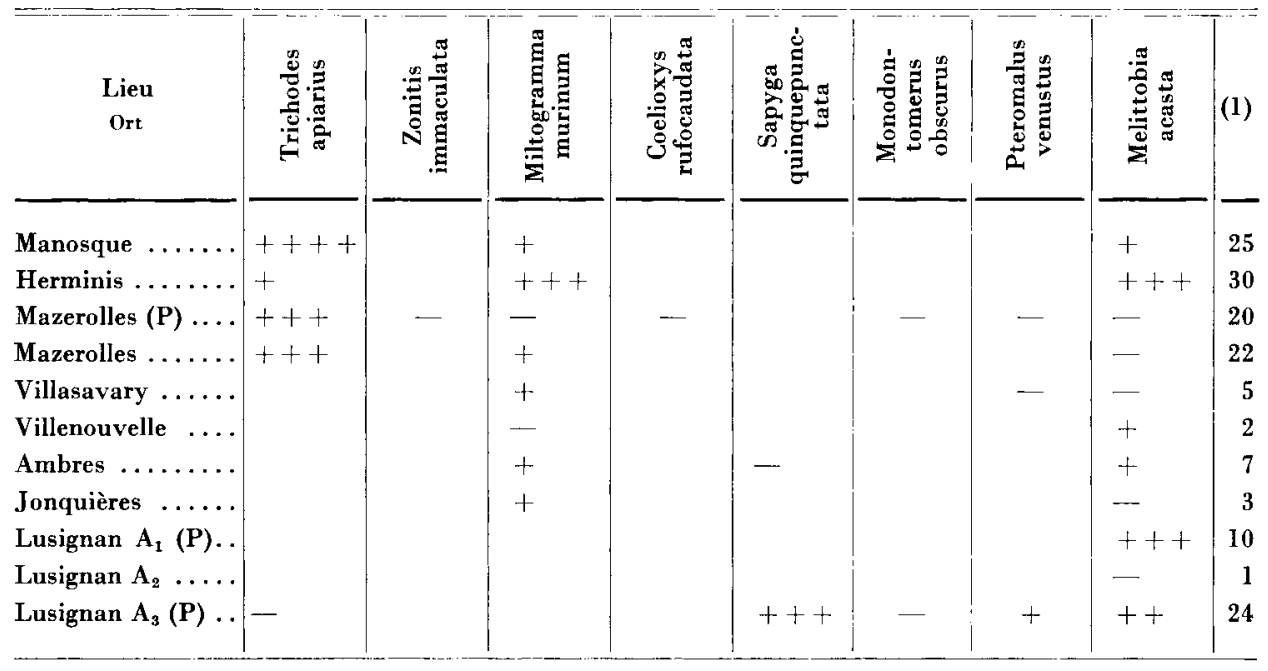

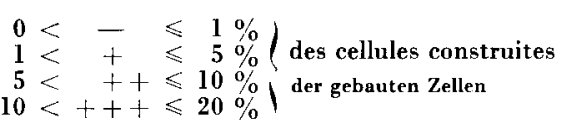

(1) Pourcentage de cellules détruites ou parasitées Prozentsatz der zerstörten oder parasitierten Zellen

\section{III. - Récapitulation des résultats de quatre années d'élevage}

Deux conclusions peuvent être tirées de la lecture du tableau n 7 :

a) L'année 1975 est la première qui permette d'accroître le stock de cellules de mégachiles. Si l'on considère l'augmentation du nombre des femelles la multiplication s'est faite en moyenne dans le rapport : 2,2 alors que les 3 années précédentes ce rapport est égal à 0,1 .

b) Quel que soit le facteur en jeu (émergence d'adultes après le retrait des nichoirs, parasitisme, mortalité pour causes indéterminées) les pertes sont les plus faibles en 1975 et au total elles n'excèdent pas 22 pour cent des cellules construites.

\section{DISCUSSION}

\section{1. - Sur la souplesse d'emploi des mégachiles}

Grâce à la dispersion des champs d'observation dans plusieurs sites géographiques bien distincts on peut constater que l'adaptation des mégachiles est relativement souple puisque la multiplication a été possible même 
TABL. 7. - Résultats des êlevages de Megachile pacifica de 1972 à 1975.

ТАв. 7. - Aufzuchtergebnisse von Megachile pacifica in den Jahren 1972-1975.

\begin{tabular}{|c|c|c|c|c|c|}
\hline \multicolumn{2}{|c|}{$\begin{array}{c}\text { Année } \\
\text { Jahr }\end{array}$} & \multirow{2}{*}{$\begin{array}{c}1972 \\
2\end{array}$} & \multirow{2}{*}{$\begin{array}{c}1973 \\
3\end{array}$} & \multirow{2}{*}{$\frac{1974}{4}$} & \multirow{2}{*}{1975} \\
\hline $\begin{array}{l}\text { Nombre de site } \\
\text { Anzahl d. Plätze }\end{array}$ & $\ldots \ldots$ & & & & \\
\hline & Région & $\begin{array}{l}\text { Sud-Est } \\
\text { Süd-Ost }\end{array}$ & $\begin{array}{l}\text { Sud-Est } \\
\text { Sid-Ost }\end{array}$ & $\begin{array}{l}\text { Sud-Est } \\
\text { Süd-Ost }\end{array}$ & $\begin{array}{l}\text { Sud-Est } \\
+ \text { Sud } \\
+ \text { Centre- } \\
\text { Ouest } \\
\text { Süd-Ost + Süd } \\
+ \text { Mittel-West }\end{array}$ \\
\hline \multicolumn{2}{|c|}{$\begin{array}{l}\text { Nombre de cellules « parents } » \ldots \ldots \ldots \ldots \\
\text { Zahl d. « Eltern } \text { "Zellen }\end{array}$} & 11000 & 12150 & 8220 & 23664 \\
\hline \multicolumn{2}{|c|}{$\begin{array}{l}\text { Nombre de cellules construites " filles » .... } \\
\text { Zahl d. gebauten "Tochter "-Zellen }\end{array}$} & 9890 & 4960 & 3035 & 40248 \\
\hline \multicolumn{2}{|c|}{$\begin{array}{l}\text { Nombre de larves saines } \ldots \ldots \ldots \ldots \ldots \ldots \\
\text { Zahl d, gesunden Maden }\end{array}$} & 2140 & 2245 & 928 & 30862 \\
\hline 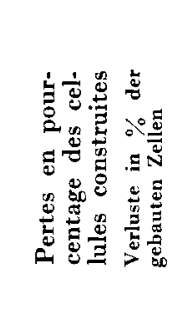 & $\begin{array}{l}\text { Emergence d'adultes } \ldots \ldots \\
\text { Schlupf } d \text {, Adulten } \\
\text { Larves mortes ........ } \\
\text { Abgestorbene Maden } \\
\text { Larves dévorées } \ldots \ldots \ldots \\
\text { Vernichtete Maden } \\
\text { Total } \ldots \ldots \ldots \ldots \ldots \\
\text { Insgesant }\end{array}$ & $\begin{array}{l}25,8 \\
78,4\end{array}$ & $\begin{array}{l}15,6 \\
54,7\end{array}$ & $\begin{array}{l}32,5 \\
78,4\end{array}$ & $\begin{array}{l}12,4 \\
21,9\end{array}$ \\
\hline 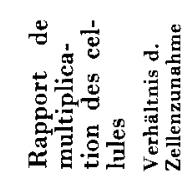 & $\begin{array}{l}\text { Avant calcul des pertes.. } \\
\text { Vor Abzug d. Verluste } \\
\text { Après calcul des pertes .. } \\
\text { Nach Abzug d. Verluste }\end{array}$ & $\begin{array}{c}0,9 \\
(0,6-1,3) \\
0,2 \\
(0,1-0,2)\end{array}$ & $\begin{array}{c}0,4 \\
(0,2-1,2) \\
\\
0,2 \\
(0-0,7)\end{array}$ & $\begin{array}{c}0,4 \\
(0,1-0,7) \\
0,1 \\
(0-0,2)\end{array}$ & $\begin{array}{c}1,7 \\
(0,8-2,3) \\
1,3 \\
(0,5-2,0)\end{array}$ \\
\hline \multicolumn{2}{|c|}{$\begin{array}{l}\text { Rapport de multiplication des femelles ..... } \\
\text { Verhältnis der Zunabme an Weibchen }\end{array}$} & 0,1 & 0,1 & 0,1 & $\begin{array}{l}2,2 \\
(0,4-3,4)\end{array}$ \\
\hline
\end{tabular}

en zone tempérée (Centre-Ouest) où le nombre journalier moyen d'heures d'activité est la moitié de celui qui est observé en zone méridionale chaude.

L'utilisation de l'espèce est facilitée par la technique consistant à laisser l'incubation se terminer dans les conditions naturelles. En effet aucun dommage n'est à craindre et de plus on note une tendance à la synchronisation 
des sorties de mégachiles et de la floraison, qui est particulièrement intéressante lorsqu'une période fraîche vient retarder la floraison.

\section{2. - Sur la longueur de la période totale de nidification}

Bien qu'appauvrie par l'été sec de 1975 la flore spontanée se révèle suffisante pour que la deuxième génération survive et construise dans la plupart des champs d'expérience. De plus les températures anormalement basses en fin de saison ont entravé l'activité des femelles, retardant le terme de l'élevage, mais au total les pertes sont modérées malgré des conditions dures.

L'allongement de 2 mois de la période de nidification, dû à l'élevage dans le champ de la deuxième génération accroît le risque de multiplication de parasites (Melittobia) et permet aux prédateurs (Trichodes) qui se sont introduits dans les nids en juillet, de poursuivre leur travail destructeur. Ce risque, ainsi que la possibilité d'avoir une fin d'été fraîche ou pauvre en sources de nectar et pollen plaident en faveur de mégachiles à génération annuelle unique. C'est également l'avis de Torchio (communication personnelle) et d'autres chercheurs étrangers.

\section{3. - Sur l'attractivité des nichoirs}

Les élevages de 1975 confirment certaines observations effectuées en 1974 sur l'attractivité de divers types de nichoirs.

a) Les trous de $5 \mathrm{~mm}$ de diamètre sont plus attractifs que ceux de $6 \mathrm{~mm}$ sans pour autant réduire de façon significative la taille de l'abdomen des femelles. Les mégachiles s'adaptent à la cavité en la tapissant d'un plus ou moins grand nombre de morceaux de feuille : 7 dans le premier cas, 9 dans le second.

b) La fixation d'un fort pourcentage de femelles sur les nichoirs après leur émergence est certainement due en grande partie à la contiguïté du bord postérieur des plaques cannelées et $d u$ fond du nichoir. L'importance de ce détail de construction m'a été confirmée par Manninger et Torchio (Communications personnelles).

\section{4. - Sur l'intérêt des abris en polyane}

De tels abris ont été employés il y a une dizaine d'années par les auteurs canadiens Pankiw et Siemens (1974) qui les recommandent dans les zones tempérées du Canada où il n'y aurait sans cet artifice aucune possibilité de multiplier les mégachiles. Des essais 1975 , je ne prétends tirer aucune conclusion définitive quant à leur intérêt dans notre pays, tout au plus quelques 
remarques : Bien qu'il ne soit pas indispensable sous notre climat, particulièrement dans le sud de la France, l'abri en poly ane crée un microclimat plus chaud que celui du simple abri en bois, d'où certains effets avantageux qui ont été perçus de façon sensible à Lusignan c'est-à-dire en zone tempérée :

- La réduction de la fin de la période d'incubation en champ donc le regroupement des émergences.

- L'augmentation de l'attractivité du site pour les femelles $(+25$ à +50 pour cent).

- L'augmentation de l'activité de construction des femelles $(+30$ pour cent).

- La réduction des délais d'émergence des individus de deuxième génération, done la réduction de la période totale d'activité des abeilles.

Cependant on note un accroissement du pourcentage de nids de première génération donnant une deuxième génération, ce qui augmente les risques de pertes en cas de mauvais temps fin août. Par ailleurs le microclimat sous polyane semble favoriser la multiplication des parasites à cycle court (Melittobia).

\section{Conctusions}

1. L'année 1975 apporte la preuve qu'avec des techniques assez simples et dans des conditions climatiques qui ne sont pas exceptionnellement favorables il est possible d'obtenir une multiplication de la souche américaine de Megachile pacifica.

2. Les techniques d'élevage doivent être encore améliorées en particulier grâce à l'emploi d'abris en polyane.

3. Le développement de tels élevages pourrait être dans l'avenir entravé par 2 problèmes sur lesquels il convient d'attirer l'attention :

a) L'émergence assez importante d'une deuxième génération après la floraison de la luzerne qui oblige l'éleveur à maintenir son abri dans le champ en août et septembre dans l'espoir d'accroître son cheptel, mais avec le risque d'en voir la diminution du fait des variations climatiques et de la multiplication de parasites polyvoltins.

b) La possibilité de “ recycler » d'une année à l'autre des parasites qui ne sont pas piégés en étuve par les rayons ultra-violets lors de la réactivation.

En conséquence deux orientations prioritaires de travail se dessinent :

- La recherche d'une lignée monovoltine de mégachiles, ce qui conduira à s'intéresser de près à l'écotype français monovoltin et à la génétique de la diapause. 
- L'étude de la dynamique des populations des parasites principaux dans les conditions bien spéciales de l'élevage artificiel. Ceci implique des travaux sur la biologie et le comportenıent des principales espèces de parasites et de cleptoparasites.

Reçu pour publication en octobre 1976.

Eingegangen im Oktober 1976.

\section{REMERCIEMENTS}

Je remercie la Fédération Nationale des Agriculteurs Multiplicateurs de Semences et l'Institut Technique des Cultures Fourragères pour son appui financier et technique pendant la période estivale.

\section{ZUSAMMENFASSUNG}

Während dreier aufeinanderfolgender Jahre wurden in Frankreich verschiedene Aufzuchttechniken an Megachile pacifica (Panz.) untersucht. Dazu wurden die Reaktionen dieser Art in Nistanlagen analysiert, die im Südosten Frankreichs aufgestellt worden waren (TASEI, 1973). Ziel des vierten Untersuchungsjahres was es, in elf gleichartigen Nistgelegenheiten die günstigsten Aufzuchtverfahren zur Vermehrung von Megachile herauszufinden.

\section{Material und Methode}

1975 wurden elf Versuchsanlagen in den Luzernefeldern an acht verschiedenen Orten, die aus Abb. 1 ersichtlich sind, aufgestellt und zwar verteilt auf neun Felder, deren Daten in Tab. 1 angegeben sind. Die Standardvorrichtung (Abb. 2) besteht aus einem gelb gestrichenen Sperrholzkasten mit schrägem Dach $(0,7 \times 0,55 \times 0,3 \mathrm{~m})$, dessen seitliche Metallständer mit Seilen verspannt sind. Jedes Schutzhäuschen ist nach Süden ausgerichtet. Im Kasteninnern befinden sich 1. eine Nistanlage aus gerieften Kiefernholzplatten, die 880 Nester von $5 \mathrm{~mm}$ Durchmesser und $8 \mathrm{~cm}$ Länge enthalten; als Rückwand ist den Nestern eine mit einer Gummischicht beklebte Holzplatte aufgeschraubt; 2. ein Schlüpfraum für die " Elternzellen "; 3. ein Nest für Trichodes, das aus einer losen Anhäufung von grobgerieften $(8 \mathrm{~mm})$ Holzplättchen besteht, die mit einer syrupartigen Mischung aus Pollen und Zuckerwasser gefüllt werden. Eine Abwandlung der Anlage ist ein Polyäthylen-Schutz, der über dem Sperrholzkasten angebracht ist (Abb. 3). Diese Vorrichtung wurde an drei Plätzen geprüft.

Bei konstant $29^{\circ} \mathrm{C}$ wurden 23600 aus Kanada eingeführte Zellen mit einem Geschlechtsverhältnis von 0,4 Weibchen zu 1 Männchen reaktiviert. Ausser in Lusignan, wo ungefähr nur 1900,1800 und 900 Zellen aufgestellt wurden, waren in jeder Nistanlage 2300 bis 2500 Kokons untergebracht. Die Nistanlagen blieben auf dem Feld, bis die 2. Generation den Nestbau beendet hatte.

Einmal wöchentlich wurden in jeder Nistanlage die verdeckelten Zellen gezählt. Am Ende der Saison wurden die Nistanlagen abgeräumt und die Zellen herausgenommen, sortiert und gezählt.

\section{Ergebnisse}

1. Tabelle 2 enhält die Angaben über die Zunahme der Zellen vor und nach Abzug der Verluste (Durchschnitt : 1,7 und 1,3). Wenn man das Verhältnis der Weibehen in der Nachkommenschaft in betracht zieht (Tab. 3), das durchschnittlich $46 \%$ beträgt, sind die Ergebnisse der Zunahme an Weibchen um 0,4 bis 3,4 höher $(\varnothing=2,2)$. 
2. Die klimatischen Unterschiede an den einzelnen Versuchsorten (Tab. 4) hatten die erwarteten Veränderungen der Bautätigkeit nicht zur Folge.

3. Die Dynamik der Maden-Populationen in jeder Nistanlage lässt sich in drei Stufen zerlegen (Abb. 4, 5, 6) : rasche Zunahme der Tiere im Juli; je nach Lage des Aufstellungsortes eine mehr oder minder heftige Abnahme im August und ein neues Anwachsen der Zahlen im September. Auf Grund der niedrigen Temperaturen Ende August und im September ist die Population am Ende der Saison nicht mehr so stark wie Ende Juli.

4. An acht von elf Plätzen war die Rate der zu den Schlüpfplätzen zurückkehrenden Weibchen höher als $50 \%$.

5. Die zweite Generation war überaus zahlreich, sie umfasste 44 bis $77 \%$ der während der Flugzeit der 1. Generation gebauten Zellen.

6. Durchschnittlich baute ein Weibchen der 1. Generation 15 bis 20 Zellen, ein Weibchen der 2. Generation dagegen auf Grund der ungünstigen Wetterverhältnisse am Ende des Sommers 1975 nur 2 bis 3 Zellen. Trotz der für die Jahreszeit zu niedrigen Temperaturen (Maximum 22 bis $23{ }^{\circ} \mathrm{C}$ ) im Mittelwesten wurde eine gewisse Nestbautätigkeit aufrechterhalten (Abb. 7).

7. Nach der Blütezeit der Luzerne sind die Pflanzen, auf denen Megachile sammeln kann, nicht sehr zahlreich. Sie gehören zu den Kompositen (Inula, Helminthia, Picris), den Rosaceen (Rubus), den Papilionaceen (Lotus), den Cruciferen und Umbelliferen (Abb. 5).

8. Die drei Hauptfeinde der Megachile-Nester sind : Trichodes apiarius L., Miltogramma murinum Mg. und Melittobia acasta Walk.. Die von diesen Insekten verursachten Schäden betreffen 1 bis $25 \%$ der Zellen. Das durch andere Faktoren verursachte Absterben umfasst 10 bis $17 \%$ der Zellen.

9. Die Polyäthylen-Schutzhäuschen scheinen die Attraktivität der Nistanlagen zu erhöhen und in geringerem Umfang auch die Tätigkeit der Weibchen zu beeinflussen. Ausserdem erlaubt das von ihnen gewährleistete warme Mikroklima eine Umschichtung des Schlüpfens und beschleunigt den Ausflug der Adulten der 2. Generation. Ein Nachteil dieser Vorrichtung dürfte die Zunahme der Zellen der 2. Generation sein; und sie künnte die Vermehrung der Parasiten mit kurzem Zyklus begünstigen (Melittobia).

10. Die weiteren Untersuchungen müssen auf die Verbesserung der Techniken, das Studium der Diapause, des französischen, monovoltinen Ökotyps sowie der Dynamik der Parasiten-Populationen ausgerichtet werden.

\section{RÉFÉRENCES BIBLIOGRAPHIQUES}

Anonyme, 1972, Programme de pollinisation (en espagnol). Gaceta del Colmenar 34, 78-79.

Bohart G. E., 1962, How to manage the alfalfa leaf-cutting bee (Megachile rotundata F.) for alfalfa pollination. Circ. 144 Agr. Exp. Sta. Logan. 7 p.

Ciundarescu G., Varga P., 1975, Preliminary research on the acclimatization of the alfalfa pollinator the wild bee (Megachile rotundata Fabr.) in the Romanian ecology, An I.C.C.P.T. 40, 355-359.

Hоввs G. A., 1965, Importing and managing the alfalfa leafcutter bee. Can. Dep. Agric. Publ. 1209, 11 p. 
Manninger S., 1975, Megachile rotundata. Son utilisation à grande échelle et sa production de masse en Hongrie. C.R. III Symp. Int. Pollinisation. Prague 1974, 261-265.

Manninger S., Communication personnelle.

Pankiw P., Siemens B., 1974, Management of Megachile rotundata in Northwestern Canada for population increase. Can. Entomol. 106, 1003-1008.

Ptacek V., 1975. Notes sur l'élevage de Megachile rotundata en Tchécoslovaquie. C.R. III Symp. Int. Pollinisation. Prague 1974, 267-273.

TAsei J.-N., 1975, Le problème de l'adaptation de Megachile (Eutricharaea) pacifica Panz. (Megachilidae) américain en France. Apidologie 6, 1-57.

Torchro P. F., Communication personnelle. 